\title{
$\zeta$ Factor and k-Factor Determination Using Needle Samples
}

\author{
Hendrik O. Colijn ${ }^{1}$, David W. McComb ${ }^{1}$ \\ ${ }^{1}$ Center for Microscopy and Analysis, The Ohio State University, Columbus, OH, USA.
}

The Cliff-Lorimer method for TEM/STEM EDX analysis is the most common method of EDX quantification. [1] While most researchers use k-factors supplied by the detector manufacturer, it is generally preferable to use experimentally determined standards. Acquiring appropriate multi-element standards can be challenging since these standards need to be homogeneous on a small scale with no surface films. Once adequate standards have been obtained, it is still necessary to account for absorption in the sample.

The $\zeta$ factor method [2] is useful if the sample and holder geometry is well defined and a beam current monitor is available. Few TEMs, though, are equipped with accurate beam current monitors. Also, on many TEMs, there can be a difference in collection efficiency across the sample. For example, on a standard 3-post Omniprobe ${ }^{\mathrm{TM}}$ grid held in an FEI double-tilt cradle, the shadowing or "penumbra" [3] of the holder can vary from 7 to 14 degrees depending on the pillar used. The effect on the $\zeta$ factor is illustrated in Table 1. The advantage of the $\mathrm{k}$-factor technique is that the end user need not measure the beam current accurately (or at all). Also the k-factor technique is not sensitive to the location of the beam on the sample, though it is sensitive to the sample thickness. While the $\zeta$ method can use pure element standards, most k-factors are determined using multi-element standards. This adds additional uncertainty to the result.

An approach combining $\zeta$ and $\mathrm{k}$ factors overcomes some of the problems associated with the individual techniques. Taking the ratio of the $\zeta$ factors produces the $\mathrm{k}$-factors. Using pure element standards removes the concern about the stoichiometry of the standard. Only the microscope that is preparing the $\zeta$ factors need be equipped with a Faraday cup.

Both the $\mathrm{k}$-factor and $\zeta$ factor methods require a measurement of sample thickness. FIB needle samples are ideal since the circular cross-section means that the diameter and thickness are the same. The taper on the FIB needle samples also allows multiple measurements at different known thicknesses. By making multiple measurements the uncertainty in the $\zeta$ factor can be estimated. This uncertainty can then be carried through the calculations for the k-factors. The k-factor uncertainty can then be added into the total uncertainty budget of the measurement.

We prepared needle samples from pure elements, mounted the needles in a fixed geometry and collected the spectra using a Tecnai F20 with a $30 \mathrm{~mm}^{2}$ EDAX XLT detector. The probe was stepped along the centerline of the needle and the diameter was measured at each step while monitoring the current with a Faraday cup. The resulting $\zeta$ factors were then averaged and the experimental uncertainty determined. From this information we calculate the absorption-free k-factors relative to $\mathrm{Si}$ and the uncertainties as shown in Table 2. As with all k-factor calculations, absorption corrections should be included for accurate quantification. [5] 


\section{References:}

[1] see e.g. D.B. Williams \& C.B. Carter, Transmission Electron Microscopy: A Textbook for Materials Science, (Springer), 2009, chapter 35.

[2] M. Watanabe, D.B. Williams, Journal of Microscopy, 221 pt2, p89 (2006).

[3] N.J. Zaluzec, Microscopy \& Microanalysis, 22 (Suppl 3), 2016, p. 278 (2016).

[4] Colijn, Hendrik O., McComb, David W., k-factor and ל-Factor determination for TEM SDD Detectors With Large Solid Angles; Materials Science \& Technology (2015) F20.

[5] The authors acknowledge support from The Ohio State University and the Ohio Third Frontier Research Scholar program.

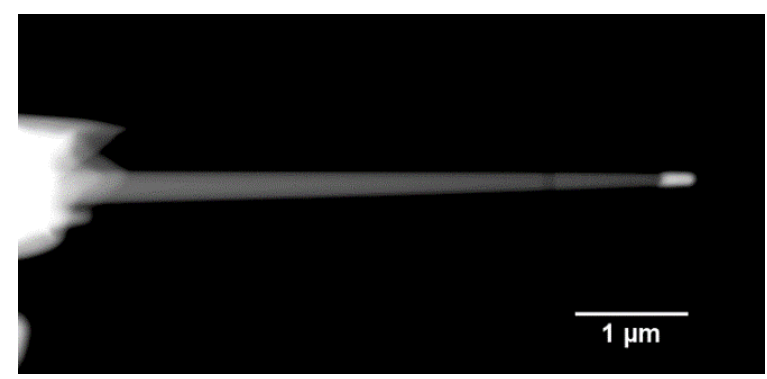

Figure 1. Cross-sectional needle sample of Si with an oxide layer and an Al coating.

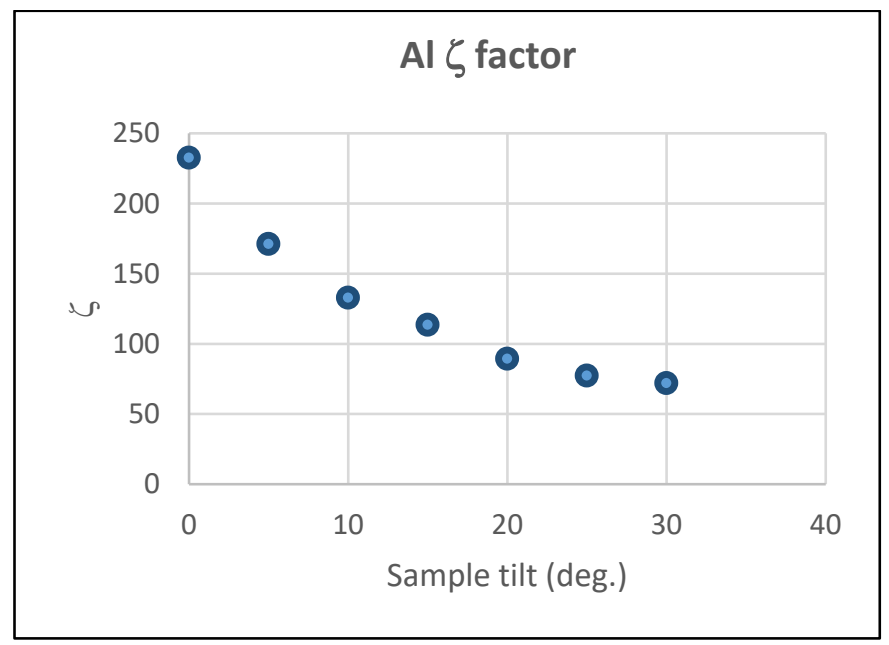

\begin{tabular}{|c|c|}
\hline Position 1 & Position 2 \\
\hline 114 & 124 \\
\hline
\end{tabular}

Table 1. $-\mathrm{Si} \zeta$ factors from different OmniProbe ${ }^{\mathrm{TM}}$ grid posts.

Figure 2. $\mathrm{Al} \zeta$ factor as a showing the effect of the sample rod penumbra.

\begin{tabular}{|c|c|c|c|c|c|c|c|c|}
\hline & & & & \multicolumn{2}{|c|}{ atomic } & at.wt. & \multicolumn{2}{c|}{ weight } \\
\hline & $\zeta$ & $\sigma$ & $\mathrm{rel} \sigma$ & $\mathrm{k}$ & $\sigma$ & & $\mathrm{k}$ & $\sigma$ \\
\hline $\mathrm{SiK} \alpha$ & 76.79 & 0.44 & 0.00568 & 1 & & 28.0855 & 1 & \\
\hline $\mathrm{AlK} \alpha$ & 73.60 & 1.04 & 0.01408 & 0.9584 & 0.0146 & 26.9815 & 0.921 & 0.014 \\
\hline $\mathrm{AgK} \alpha$ & 3241 & 111 & 0.03425 & 42.2039 & 1.4653 & 107.868 & 162.1 & 5.63 \\
\hline $\mathrm{MnK} \alpha$ & 131.3 & 1.61 & 0.01229 & 1.7102 & 0.0232 & 54.9380 & 3.34 & 0.045 \\
\hline $\mathrm{MoK} \alpha$ & 965.4 & 45.52 & 0.04715 & 12.5713 & 0.5971 & 54.9380 & 24.59 & 1.17 \\
\hline
\end{tabular}

Table 2. $\zeta$ factors for selected elements and the derived k-factors with uncertainty values. 